Schwerpunkt

Herzschr Elektrophys 2020 • 31:368-374 https://doi.org/10.1007/s00399-020-00700-z Eingegangen: 24. Mai 2020

Angenommen: 21. Juni 2020

Online publiziert: 13 . Juli 2020

(c) Springer Medizin Verlag $\mathrm{GmbH}$, ein Teil von Springer Nature 2020

\author{
Jakob Ledwoch' · David Duncker ${ }^{2}$ \\ ${ }^{1}$ Klinik für Kardiologie, Pneumologie und Internistische Intensivmedizin, München Klinik Neuperlach, \\ München, Deutschland \\ ${ }^{2}$ Rhythmologie und Elektrophysiologie, Klinik für Kardiologie und Angiologie, Medizinische Hochschule \\ Hannover, Hannover, Deutschland
}

\title{
eHealth - Smart Devices revolutionieren die Kardiologie
}

\begin{abstract}
Der Bereich eHealth erfährt eine rasante Entwicklung, und digitale Konzepte werden im Medizinsektor und in der Kardiologie zunehmend umgesetzt. Gerade Smart Devices wie Smartphones und Smartwatches spielen im eHealth-Bereich eine große Rolle, da sie von einem Großteil der Bevölkerung genutzt werden und sehr viele Anwendungsmöglichkeiten in einem Gerät bündeln. Dies führt dazu, dass auch die Industrie und die Patienten selbst den Einsatz dieser Geräte vorantreiben. Auch die großen kardiologischen Fachgesellschaften gehen davon aus, dass mobile Devices inklusive der dazugehörigen Apps bei der Kommunikation von medizinischen Informationen, Entscheidungsprozessen im klinischen Alltag und telemedizinischen Erfassen von Biosignalen entscheidende Fortschritte erzielen werden. Der folgende Beitrag zeigt, was heute schon möglich ist und welche Anwendungsmöglichkeiten in naher Zukunft zu erwarten sind.
\end{abstract}

\section{Apps der großen kardiologi- schen Fachgesellschaften}

Die großen kardiologischen Fachgesellschaften wie die Deutsche Gesellschaft für Kardiologie (DGK), die European Society of Cardiology (ESC) und die American Heart Association (AHA) bieten seit einigen Jahren eigene Apps an, die ursprünglich in erster Linie eine digitale Darstellung der jeweiligen Leitlinien auf den mobilen Geräten wie
Smartphone oder Tablet zum Ziel hatten. Die dort hinterlegten Leitlinien, die im klinischen Alltag ein deutlich einfacheres und schnelleres Nachschlagewerk bieten als online hinterlegte PDFs oder gedruckte Pocketleitlinien, sind den meisten KardiologInnen bekannt. In letzter Zeit wurde in diesen Apps vor allem der Bereich mit speziellen interaktiven Anwendungen deutlich ausgebaut. Diese Tools bieten verschiedenste Möglichkeiten, den klinischen Alltag zu vereinfachen. Beispielsweise enthält die ESC Leitlinien-App Tools zu verschiedenen Risikokalkulatoren (z. B. Risiko für plötzlichen Herztod bei hypertropher Kardiomyopathie [HCM]), "clinical decision support“ (CDS; z. B. standardisiertes Vorgehen bei Statinintoleranz) sowie Übersichtslisten (z.B. Sicherheitsprofil kardiovaskulärer Medikamente bei Schwangeren). Noch existiert keine Evidenz zum Gebrauch der Leilinien-Apps und ob sich damit die medizinische Versorgung verbessern und Arbeitsabläufe effizienter gestalten lassen. Einige Studien im Bereich CDS sind allerdings schon verfügbar und werden im Folgenden diskutiert.

\section{"Clinical decision support ${ }^{\prime \prime}$}

Aufgrund des zunehmenden Wissenszuwachses in der Medizin wird es für Ärzte immer schwerer, alle Informationen für bestimmte Fragestellungen im klinischen Alltag abrufen zu können und stets leitliniengerecht $\mathrm{zu}$ entscheiden. Helfen könnte hier ein CDS. Das sind strukturierte, meist digitale Systeme, die den klinischen Entscheidungsprozess mithilfe verschiedener Methoden wie Entscheidungsbäumen oder Frage-Antwort-Möglichkeiten unterstützen. Durch ein CDS kann nicht nur die Leitlinienadhärenz erhöht, sondern es können Kosten reduziert (Reduktion unnötiger oder wiederholter Untersuchungen, Wahl von kosteneffektiveren Behandlungen), der administrative Aufwand gesenkt (automatische DRG-Kodierung, automatisierte Dokumentation) und die Diagnostik verbessert (optimierte Empfehlung zu diagnostischen Untersuchungen, automatisierte Darstellungen diagnostischer Resultate) werden [25]. Prädestiniert für ein CDS sind Applikationen auf einem Klinikrechner oder auf einem Smartphone, welche passend zum jeweiligen medizinischen Problem direkt aufgerufen werden und den Arzt basierend auf den aktuellen Leitlinien durch den Entscheidungsprozess leiten. Der Nutzen eines solchen Vorgehens ist bereits in klinischen Studien belegt. Mithilfe von CDS-Systemen konnten beispielsweise CT-Untersuchungen bei Patienten mit Verdacht auf Lungenarterienembolien in der Notaufnahme um $20 \%$ reduziert werden [22]. Zudem wurde die Rate an korrekt positiven CTBefunden und die Leitlinienadhärenz in Bezug auf den Einsatz von CT signifikant erhöht [9]. Auch die Therapie von Herzinsuffizienzpatienten lässt sich durch einen automatisierten CDS verbessern. Ein solches System erkannte durch den automatischen Zugriff auf demographische Patienteninformationen sowie Echokardiographie- und EKG-Daten eine Verschlechterung der Herzinsuffizienz der teilnehmenden Patienten und 


\begin{tabular}{|c|c|c|}
\hline Bereich & App & Funktion \\
\hline \multirow[t]{3}{*}{$\begin{array}{l}\text { Allgemeine } \\
\text { Kardiologie }\end{array}$} & DGK Pocket-Leitlinien & $\begin{array}{l}\text { Nachschlagewerk für Leitlinien, Tools zu CDS } \\
\text { und verschiedene Kalkulatoren }\end{array}$ \\
\hline & ESC Pocket Guidelines & $\begin{array}{l}\text { Nachschlagewerk für Leitlinien, Tools zu CDS } \\
\text { und verschiedene Kalkulatoren }\end{array}$ \\
\hline & ACC Guideline Clinical App & $\begin{array}{l}\text { Nachschlagewerk für Leitlinien, Tools zu CDS } \\
\text { und verschiedene Kalkulatoren }\end{array}$ \\
\hline \multirow[t]{3}{*}{ Rhythmologie } & $\begin{array}{l}\text { EP mobile (EP Studios, Inc., } \\
\text { USA) }\end{array}$ & $\begin{array}{l}\text { CDS zur Diagnose verschiedener Arrhythmi- } \\
\text { en, Kalkulatoren, Risikoscores }\end{array}$ \\
\hline & $\begin{array}{l}\text { EP tools (Busy Being Born } \\
\text { Solutions LLC, USA) }\end{array}$ & $\begin{array}{l}\text { CDS zur Diagnose verschiedener Arrhythmi- } \\
\text { en, Kalkulatoren, Risikoscores }\end{array}$ \\
\hline & $\begin{array}{l}\text { ESC AF manager (European } \\
\text { Society of Cardiology) }\end{array}$ & $\begin{array}{l}\text { CDS zum leitliniengerechten Management } \\
\text { von Vorhofflimmerpatienten (Möglichkeit der } \\
\text { Verknüpfung mit der Patienten-App „myAF“ } \\
\text { der ESC) }\end{array}$ \\
\hline \multirow[t]{6}{*}{$\begin{array}{l}\text { Interventionelle } \\
\text { Kardiologie }\end{array}$} & $\begin{array}{l}\text { BifurcAID (Mount Sinai } \\
\text { Hospital, USA) }\end{array}$ & $\begin{array}{l}\text { CDS zu verschiedenen Interventionstechniken } \\
\text { von Bifurkationsstenosen }\end{array}$ \\
\hline & $\begin{array}{l}\text { OCTAID (Mount Sinai Hos- } \\
\text { pital, USA) }\end{array}$ & $\begin{array}{l}\text { Bildatlas verschiedener OCT-Befunde, OCT- } \\
\text { Quiz, Kommunikation mit OCT-Experten }\end{array}$ \\
\hline & $\begin{array}{l}\text { TransseptAID (Mount Sinai } \\
\text { Hospital, USA) }\end{array}$ & $\begin{array}{l}\text { CDS zur transseptalen Punktion bei verschie- } \\
\text { denen Interventionen, Materialkunde, Kom- } \\
\text { plikationsmanagement }\end{array}$ \\
\hline & $\begin{array}{l}\text { CalcificAID (Mount Sinai } \\
\text { Hospital, USA) }\end{array}$ & $\begin{array}{l}\text { CDS zu verschiedenen Interventionstechniken } \\
\text { bei verkalkten Läsionen }\end{array}$ \\
\hline & $\begin{array}{l}\text { TAVRcathAID (Mount Sinai } \\
\text { Hospital, USA) }\end{array}$ & $\begin{array}{l}\text { CDS zur Koronarangiographie bei implantier- } \\
\text { ter Aortenklappenprothese }\end{array}$ \\
\hline & $\begin{array}{l}\text { Stent\&Surgery (SICI-GISE, } \\
\text { Italien) }\end{array}$ & $\begin{array}{l}\text { CDS zur antithrombozytären Therapie nach } \\
\text { Interventionen bei Patienten vor Operation }\end{array}$ \\
\hline
\end{tabular}

Tab. 2 Sensitivität und Spezifität von verschiedenen Smartphone-Apps zur Vorhofflimmerdetektion. (Ergebnisse basierend auf der Metaanalyse von O'Sullivan et al. [19])

\begin{tabular}{|c|c|c|}
\hline App & $\begin{array}{l}\text { Sensitivität } \\
\text { (95\% Konfidenzintervall) }\end{array}$ & $\begin{array}{l}\text { Spezifität } \\
\text { (95\% Konfidenzintervall) }\end{array}$ \\
\hline Cardiio (Cardiio Inc., USA) & $93,5(89,2-96,2)$ & $94,8(88,3-97,8)$ \\
\hline $\begin{array}{l}\text { FibriCheck (Qompium, } \\
\text { Belgien) }\end{array}$ & $96,9(94,1-98,4)$ & $96,0(86,6-98,9)$ \\
\hline $\begin{array}{l}\text { Preventicus (PREVENTICUS } \\
\text { GmbH, Deutschland) }\end{array}$ & $92,9(88,1-95,8)$ & $98,7(84,3-99,9)$ \\
\hline McManus et al & $97,1(91,4-99,1)$ & $93,4(87,3-96,7)$ \\
\hline Alle & $94,2(92,2-95,7)$ & $95,8(92,4-97,7)$ \\
\hline
\end{tabular}

informierte den betreuenden Arzt mit einer automatisch generierten Email über diese Veränderung. Dies führte zu einer signifikant häufigeren Überweisung des Patienten in ein Herzinsuffizienzzentrum und schließlich zu einer niedrigeren Gesamtmortalität [10]. Im Bereich kardiovaskuläre Risikofaktoreinstellung konnte durch den Einsatz eines computerbasierten CDS-Systems die Leitlinienadhärenz der behandelnden Ärzte zur Verschreibung der notwendigen Medikamente und letztlich Erreichen
Auch die ESC und ACC erweitern ihr Tool-Angebot stetig. Für RhythmologieInteressierte bietet der AppStore Anwendungen wie EP tools (Busy Being Born Solutions LLC) oder EP mobile (EP Studios, Inc.). Weniger ein CDS im klassischen Sinn bieten diese Apps verschiedene Funktionen wie QTc-Rechner, Nachschlagewerk für Brugada- oder ARVC-Kriterien (arrhythmogene rechtsventrikuläre Kardiomyopathie), aber auch Entscheidungspfade zur Differenzierung einer Breitkomplextachykardie. Das Team der Interventionalisten des Mount Sinai Krankenhauses in New York hat eine ganze App-Sammlung erstellt. Entscheidungsprozesse zu Bifurkationsinterventionen, den Gebrauch der optischen Kohärenztomographie (OCT) oder der transseptalen Punktion werden hier unterstützt. Eine Auswahl an CDS-Tools ist in $\mathbf{0}$ Tab. 1 dargestellt. Auch im Bereich Smartphone-basierter CDS-Systeme existieren erste erfolgversprechende Daten. Die TAVIApp (TAVI center of University Magna Graecia of Catanzaro) erhöhte signifikant die Übereinstimmung hinsichtlich der Therapieplanung (Indikationsstellung, Wahl des Verfahrens, Auswahl der Prothesengröße) bei Patienten mit hochgradiger Aortenklappenstenose unter kardiologischen Assistenzärzten [13]. Die gleiche Arbeitsgruppe konnte zeigen, dass kardiologische Assistenzärzte mithilfe einer CDS-App die gleiche Rate an korrekter Klassifizierung der ESC-Empfehlungen für einen implantierbaren KardioverterDefibrillator (ICD) oder eine kardiale Resynchronisationstherapie (CRT) wie erfahrene Elektrophysiologen erzielten [7]. Auch eine Verbesserung von Therapiezielen bei der Kontrolle von kardiovaskulären Risikofaktoren konnte durch eine Smartphone-basierte App mit Implementierung von leitliniengerechter Blutdrucksenkung und Blutzuckereinstellung erzielt werden [1].

Für eine noch anwenderfreundlichere Nutzung sollten CDS-Systeme in Zukunft in die klinik- oder praxisinternen Krankenhausinformationssysteme integriert werden. Dadurch können medizinische Informationen der Patienten von den CDS-Systemen automatisch erfasst und im Hintergrund bearbei- 
tet werden. Auf diese Weise kann dem Arzt aus dokumentiertem Leitsymptom, Laborparametern und initialen Untersuchungsergebnissen, ohne diese Informationen aktiv in ein System eingeben zu müssen, eine automatisierte leitlinienorientierte Empfehlungsübersicht zum weiteren Vorgehen präsentiert werden.

\section{Erfassung von Biosignalen mit Smart Devices}

Ein großer Nutzen von Smart Devices liegt neben ihrer Funktionalität darin, dass sie von einem Großteil der Menschen in Industrienationen täglich genutzt und somit praktisch rund um die Uhr bei sich geführt werden. Mit einem Anteil der Smartphone-User von knapp $80 \%$ der Gesamtbevölkerung liegt Deutschland auf Platz 4 im weltweiten Ranking [18]. Eine derartige Penetration eines Devices in der Bevölkerung bietet ein erhebliches Potenzial, verschiedenste Daten der Anwender zu erheben. Dies schließt auch die Erfassung von medizinischen Daten (Biosignalen) mit ein. Diese Daten können genutzt werden, um die Patientenversorgung zu verbessern.

\section{Puls- und Rhythmusanalyse}

Mithilfe des Smartphones lässt sich basierend auf der Photoplethysmographie (PPG) der Puls messen. Hierfür wird ein Finger vor die Kamera und das Blitzlicht gehalten. Dabei dringt Licht aus dem Blitz in die Hautschichten des Fingers, bis es am Hämoglobin des fließenden Blutes in den Kapillaren wieder mit einer bestimmten Wellenlänge reflektiert wird. Dieses pulsatil reflektierte Lichtsignal wird von der Kamera erfasst und durch die App-Software auf dem Smartphone in eine Pulskurve transformiert (• Abb. 1). Dies ermöglicht eine Analyse der Herzfrequenz sowie der Pulsregularität und liefert somit Hinweise für das Vorliegen von Herzrhythmusstörungen, insbesondere Vorhofflimmern. Einzelne Apps zur PPG-Analyse zeigen im Hinblick auf Frequenzanalyse und Vorhofflimmererkennung eine sehr hohe Genauigkeit im Vergleich zu einem Ein-Kanal-EKG [28]. Insgesamt weisen die PPGbasierten Rhythmus-Apps eine sehr gute

Herzschr Elektrophys 2020 · 31:368-374 https://doi.org/10.1007/s00399-020-00700-z

(c) Springer Medizin Verlag GmbH, ein Teil von Springer Nature 2020

\section{J. Ledwoch · D. Duncker \\ eHealth - Smart Devices revolutionieren die Kardiologie}

\section{Zusammenfassung}

Hintergrund. Der Nutzen von Smart Devices wie Handys und Smartwatches in der Kardiologie nimmt deutlich zu. Der gehäufte Einsatz wird vor allem auch von Patienten und der Industrie vorangetrieben.

Fragestellung. Welche Möglichkeiten bieten Smart Devices in der Kardiologie?

Material und Methode. Es wurde eine selektive Literaturrecherche durchgeführt. Naturwissenschaftliche und klinische Studien über die verschiedenen Einsatzmöglichkeiten der technischen Mittel wurden interpretiert. Ergebnisse. Der Besitz und Gebrauch von Smartphones in Deutschland ist im weltweiten Vergleich sehr hoch. Dies ermöglicht einen sehr breiten Einsatz dieser Technologie auch im medizinischen Bereich. Die Anwendungsmöglichkeiten sind vielfältig und reichen von der Nutzung als Nachschlagewerk über einen "clinical decision support" bis hin zur Erfassung von Biosignalen. Gerade die Kombination aus Biosignalmessung und Weiterverarbeitung der Information durch künstliche Intelligenz (KI) ermöglicht eine deutliche Verbesserung der bisherigen Diagnosemethoden und erlaubt extrem genaue Vorhersagen verschiedener kardiovaskulärer Krankheitsverläufe. Schlussfolgerung. Smart Devices werden in der Kardiologie zunehmend im klinischen Alltag genutzt. Aufgrund der technischen Möglichkeiten wird der Einsatz sehr wahrscheinlich weiter steigen und einige Bereiche der Kardiologie deutlich verändern.

Schlüsselwörter

Kardiologie - Smartphone - Smartwatch . Wearables · Künstliche Intelligenz

\section{eHealth—smart devices revolutionizing cardiology}

\section{Abstract}

Background. The use of smart devices such as smartphones and smartwatches in cardiology continues to significantly increase. The widespread use is also promoted by patients and the industry.

Objectives. The goal is to assess different opportunities of smart devices in cardiology. Materials and methods. A selected review of the literature was carried out. Scientific and clinical studies regarding possible applications of these devices were evaluated. Results. The use of smartphones in Germany is among the highest worldwide. This allows very broad use of this technology in general and in the medical field. There are various potential applications ranging from a reference source over clinical decision support to biosignal sensing. Particularly, the combination of biosignal collection and interpretation by artificial intelligence could lead to a significant improvement of our diagnostic methods and precisely predict the course of various cardiovascular diseases. Conclusions. Smart devices are already increasingly used in daily clinical practice. Due to the numerous technical opportunities, the use of these devices will further spread and, most probably, will transform cardiology.

\section{Keywords}

Cardiology · Smartphone - Smartwatch . Wearables $\cdot$ Artificial intelligence
Sensitivität und Spezifität bei der Detektion von Vorhofflimmern auf (• Tab. 2). Aktuelle Konsensusdokumente empfehlen bereits den Einsatz derartiger neuer Devices zum Screening nach Vorhofflimmern [15].

Alternativ zur Fingermethode kann das photoplethysmographische Signal auch über eine Kameraaufnahme des Gesichts erfasst werden [31]. Hierbei detektiert die Kamera feine Schlag-fürSchlag-Variationen der Hautfarbe, die mit arteriellen Blutpulsationen korrelie- ren und somit zur Rhythmusanalyse herangezogen werden können (• Abb. 2). Da PPG-Messungen mit der Smartphone-Kamera die aktive Durchführung einer Messung durch den Anwender erfordern, können paroxysmale oder asymptomatische Arrhythmien mit dieser Methode unerkannt bleiben. Mithilfe von Devices, die vom Nutzer permanent getragen werden, wie Fitnessarmbänder, Ringe, Halsketten oder Smartwatches lässt sich ein kontinuierliches PPGMonitoring gewährleisten und so par- 


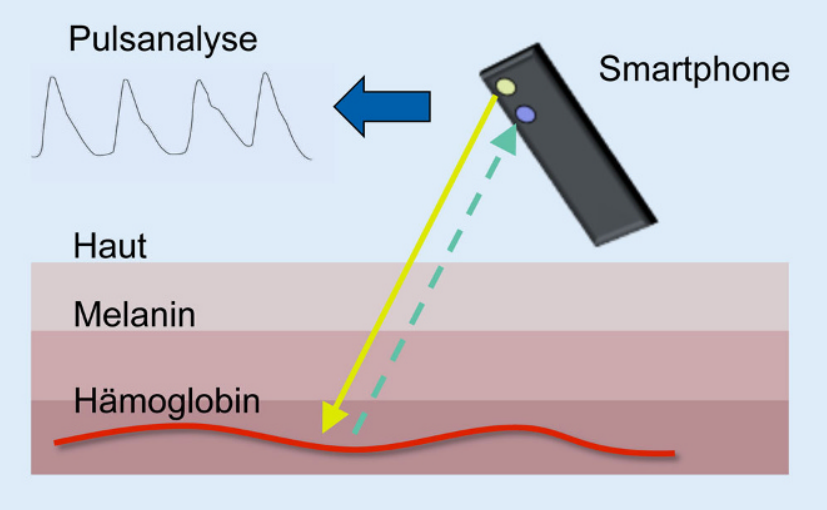

Abb. 1 A Pulsanalyse mithilfe der Photoplethysmographie. Licht aus der Lichtquelle (gelber Pfeil) des Smartphones (Blitz) gelangt durch verschiedene Hautschichten, bis es am Hämoglobin des pulsatil fließenden Blutes reflektiert wird. Das pulsatile optische Signal (grüner Pfeil) einer bestimmten Wellenlänge wird von der Smartphone-Kamera detektiert. Die optische Information kann aufgrund der Pulsatilität durch die Smartphone-Software in eine Pulskurve transformiert werden

oxysmale oder asymptomatische Arrhythmien detektieren. Bezüglich der Smartwatch-basierten PPG-Analyse zur Vorhofflimmerdetektion wurden bereits einige Daten publiziert. Ähnlich wie beim Smartphone zeichnet sich diese durch eine hohe Sensitivität und Spezifität aus, was die Ergebnisse der WATCHAF-Studie nachweisen konnten. Hier zeigte sich bei der Vorhofflimmerdetektion eine Sensitivität von $93,7 \%$ und Spezifität von 98,2\% [8]. Aufgrund ihres Studiendesigns konnten Sensitivität und Spezifität der Smartwatches in den beiden großen industriegesponsorten Studien (Apple Heart Study mit 419.297 Studienteilnehmern und Huawei Heart Study mit 246.541 Studienteilnehmern) nicht ermittelt werden. Der positive prädiktive Wert der PPG-Methode zur Vorhofflimmerdetektion lag in diesen Studien bei der Apple-Heart-Studie bei $84 \%$ [20] und bei der Huawei-Studie bei $92 \%$ [12].

Einige Smartwatches besitzen auch eine integrierte 1-Kanal-EKG-Funktion (entspricht der Ableitung I). Bei Alarmierung über eine Pulsirregularität kann mit dieser Funktion ein mögliches Vorhofflimmern verifiziert werden. Forscher an der Cleveland Clinic stellten allerdings bei 50 Patienten mit 300 EKGAufzeichnungen (erhoben mit einer Apple Watch) fest, dass der automatische Diagnosealgorithmus, der am Ende der EKG-Aufzeichnung das Resultat auf dem Bildschirm der Uhr anzeigt, zwar eine
Spezifität von $100 \%$, aber eine Sensitivität von lediglich $41 \%$ aufweist. Hauptproblem war die hohe Rate an uneindeutigen Resultaten (31\%). Erst die Interpretation der PDF-Ausdrucke des 1-Kanal-EKGs durch einen Kardiologen ergab in dieser Studie eine Sensitivität von $96 \%$ [23]. Technische Fortschritte im Diagnosealgorithmus sind hier demnach zwingend notwendig, um die Implementierung der mobilen EKG-Methode in unseren klinischen Alltag zu ermöglichen. Mit Spannung wird die von Apple initiierte Heartline-Studie erwartet, die 180.000 Patienten einschließen wird und das Vorhofflimmerscreening durch die Apple Watch mit ihrer PPG- und EKGFunktion mit einem Standardvorgehen vergleicht (NCT04276441).

\section{Blutdruck und Sauerstoffsättigung}

Basierend auf der PPG-Methode lassen sich aus der Pulskurve auch eine Blutdruckkurve und die Sauerstoffsättigung ableiten [2]. Luo et al. zeigten, dass man aus dem photoplethysmographisch ermittelten Blutfluss des Gesichts per Smartphone-Kamera eine Blutdruckkurve transformieren kann. Die Genauigkeit der Messung für den systolischen und den diastolischen Blutdruck war mit jeweils 95 und $96 \%$ sehr hoch [14]. Eine Implementierung der Blutdruckmessfunktion in eine App ist für den chinesischen Markt geplant. Wann dieses
Feature auch in Europa verfügbar sein wird, ist noch unklar.

Mithilfe der PPG-Methode lässt sich mit den Smart Devices auch die Sauerstoffsättigung messen. Eine PPG-App auf dem Smartphone zeigte in einer Studie an 81 Patienten eine sehr hohe Korrelation mit der standardmäßig pulsoximetrisch erhobenen Sauerstoffsättigung [27]. Laut Herstellerangabe können auch die ersten Smartwatches mithilfe der PPG-Methode die Sauerstoffsättigung im Blut messen. Die Datenlage auf diesem Gebiet ist allerdings noch dünn. Studien zur Vorhersage von Exazerbationen einer chronisch-obstruktiven Lungenerkrankung (NCT03857061) oder Schlafapnoe-Screening (NCT04234828; NCT03775291) basierend auf einer Smartwatch-ermittelten Sauerstoffsättigung werden aktuell durchgeführt. Die durch die Smartwatch detektierbaren Biosignale einschließlich der Sauerstoffsättigung erlauben möglicherweise auch ein Monitoring von COVID-19-Patienten. Forscher aus Heidelberg (NCT04376853) und Bern (NCT04357834) untersuchen aktuell in klinischen Studien, ob eine Verschlechterung des klinischen Zustands bei COVID-19 durch eine Smartwatch detektiert werden kann. Mit einem Smartwatch-Monitoring, das nur Herzfrequenz und Schlafzeit aufzeichnete, ließ sich bereits in einer Studie an knapp 50.000 Teilnehmern in den USA ein Vorhersagemodell erstellen, das die Verbreitung von Influenza wiedergab [21].

\section{Neue Parameter}

Neben den genannten klassischen Biosignalen können auch neuartige Parameter mithilfe der Smart Devices erfasst werden. Ein sehr simpler und weit verbreiteter Parameter ist die tägliche Schrittzahl, die nahezu durch jedes Smartphone automatisch erfasst wird. Werhahn et al. konnten zeigen, dass die per Smartphone ermittelte tägliche Schrittzahl mit der Gehstrecke im 6-Minuten-Gehtest, der maximalen Sauerstoffaufnahme und sogar der linksventrikulären Ejektionsfraktion bei Patienten mit Herzinsuffizienz korreliert [30]. Der tägliche Schrittzähler wurde auch als Parame- 

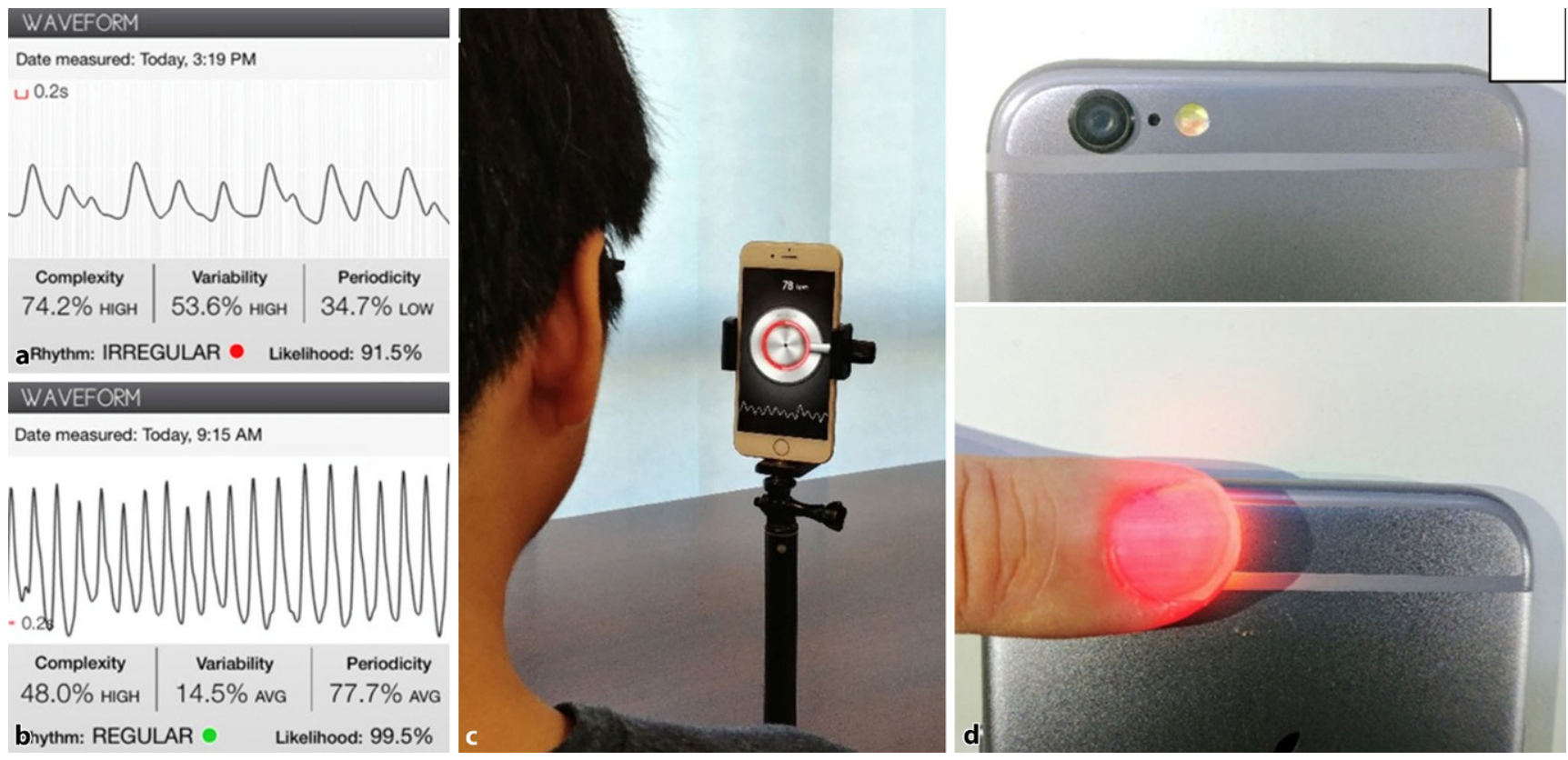

Abb. 2 A Pulsmessung mithilfe der Photoplethysmographie des Fingers und des Gesichts. a, b Rhythmusanalyse der Cardiio App. Die Detektion des optischen Signals kann aus dem Gesicht (c) oder aus dem Finger (d) erfolgen. (Aus [31])

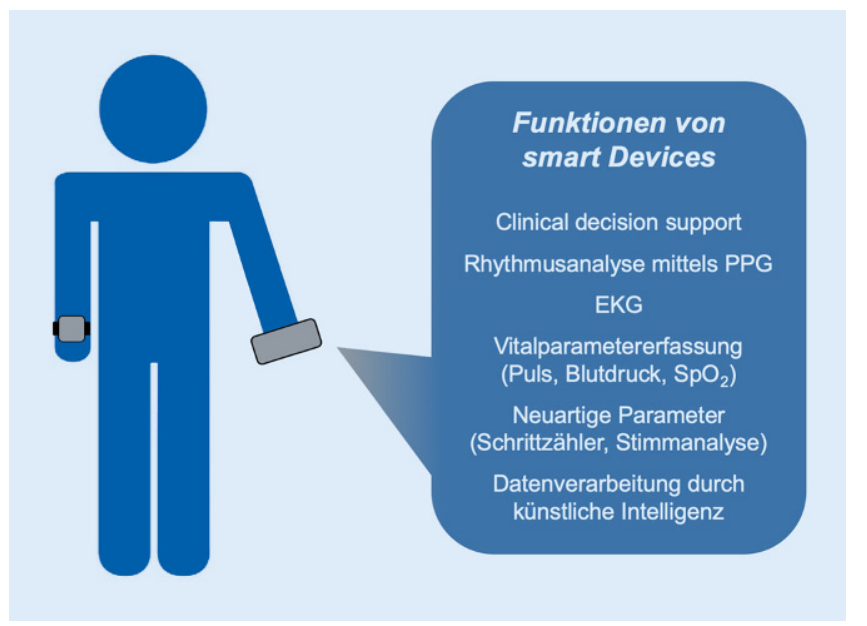

Abb. $3<$ Zusammenfassende Übersicht der medizinischen Funktionen von Smart Devices. (PPG Photoplethysmographie) ter zur Beurteilung der Belastbarkeit in einem kardiovaskulären Telerehabilitationsprojekt eingesetzt [26]. Eine andere innovative Methode zum telemedizinischen Monitoring bietet die automatisierte Stimmanalyse von Patienten. In einer Studie mit über 10.000 Patienten waren automatisch erfasste Stimmmuster bei Patienten mit Herzinsuffizienz mit kardialen Dekompensationen und der Gesamtmortalität innerhalb von 20 Monaten nach der Stimmaufzeichnung assoziiert [17]. Forscher an der Mayo-Klinik in Rochester konnten sogar mithilfe einer Smartphone-basierten Stimmaufzeichnung das Vorliegen einer koronaren Herzerkrankung voraussagen [16]. Sog. Smart Speaker sind mittlerweile in der Lage, mit Hilfe spezieller Algorithmen kontaktlos Atemmuster des Anwenders bei zentraler oder obstruktiver Apnoe $\mathrm{zu}$ analysieren und sogar eine agonale Atmung im Rahmen eines Herzstillstands zu detektieren [6].

\section{Künstliche Intelligenz}

Die Entwicklung neuer Methoden zur Detektion von verschiedenen Biosignalen wird ergänzt durch neue Methoden zur Verarbeitung dieser Signale. Künstliche Intelligenz (KI) bietet dabei einen revolutionären Ansatz und ermöglicht die Interpretation großer und komplexer Datenmengen. Ein geeignetes Einsatzgebiet von KI bietet das EKG. Der Algorithmus der Apple Watch zur Vorhofflimmerdiagnosestellung zeigt, wie bereits oben beschrieben, einige Schwächen. KI kann die diagnostische Sicherheit hier entscheidend verbessern. Durch den Einsatz von KI erzielte man in einer Studie bei der Vorhofflimmerdiagnose aus durch eine Apple Watch geschriebenen EKGs eine Sensitivität von $97,5 \%$ und Spezifität von $100 \%$ [29]. Auch die Problematik bei der Detektion von paroxysmalen oder asymptomatischen Arrhythmien lässt sich möglicherweise durch KI lösen. In einer Studie an 180.000 Patienten mit paroxysmalem Vorhofflimmern gelang es, aus ausschließlich im Sinusrhythmus erfassten EKGs Vorhofflimmern mit einer Sensitivität und Spezifität von $79 \% \mathrm{zu}$ diagnostizieren [4]. Durch eine KI-basierte EKG-Befundung lassen sich nicht nur Vorhersagen über den Rhythmus treffen. Mithilfe eines Vektorkardiogramms, basierend auf 5 durch KI interpretierte EKG-Ableitungen, ließ sich in einer Studie bei knapp $600 \mathrm{~Pa}$ tienten eine koronare Herzerkrankung mit einer Sensitivität von über $90 \%$ pro- 
gnostizieren [5]. EKG und KI erlauben aber auch Annahmen über eine Herzinsuffizienz. In einer Studie an knapp 45.000 Patienten zeigte sich, dass mit einer KI-basierten EKG-Analyse eine Herzinsuffizienz mit einer Sensitivität von $93 \%$ und Spezifität von $86 \%$ diagnostiziert werden konnte [3]. Es gelang sogar, das Risiko für eine erst Jahre später entstandene Herzinsuffizienz bei Patienten zu ermitteln, die zum Zeitpunkt der EKG-Aufzeichnung noch eine normale Pumpfunktion aufwiesen [3]. Mithilfe von Informationen aus einem Brustpatch, der neben EKG auch Temperatur, Hautimpedanz und Bewegung aufzeichnet, konnte in einer Studie an Herzinsuffizienzpatienten eine kardiale Dekompensation mit einem zeitlichen Vorlauf von knapp 7 Tagen vorhersagt werden [24]. Solch ein präziser Blick in die Zukunft erlaubt eine rechtzeitige medizinische Intervention, um zukünftige kardiale Dekompensation in diesem Patientenkollektiv zu verhindern.

Eine zusammenfassende Übersicht über die medizinischen Funktionen von Smart Devices ist in $\bullet$ Abb. $\mathbf{3}$ dargestellt.

\section{Fazit für die Praxis}

\section{- Die Entwicklung medizinischer Anwendungsmöglichkeiten der Smart Devices schreitet schnell voran. \\ - Bereits jetzt besitzen Smartphones und Smartwatches verschiedene medizinische Funktionen wie "clinical decision support" (CDS), Erfassen von Vitalparametern, Rhythmusanalyse, EKG-Aufzeichnung und die Erhebung neuartiger Parameter. \\ - In Zukunft wird die Kardiologie insbe- sondere durch die telemedizinische Erfassung von Biosignalen mithilfe von Wearables und die weitere Ver- arbeitung der Informationen durch künstliche Intelligenz (KI) revolutio- niert werden.}

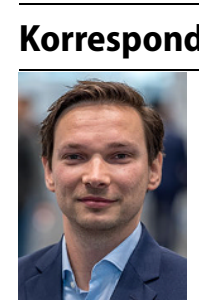
Klinik für Kardiologie, Pneumologie und Internistische Intensivmedizin, München Klinik Neuperlach

Oskar-Maria-Graf-Ring 51, 81737 München, Deutschland jakobledwoch@yahoo.de

\section{Einhaltung ethischer Richtlinien}

Interessenkonflikt. J. Ledwoch und D. Duncker geben an, dass kein Interessenkonflikt besteht.

Für diesen Beitrag wurden von den Autoren keine Studien an Menschen oder Tieren durchgeführt. Für die aufgeführten Studien gelten die jeweils dort angegebenen ethischen Richtlinien.

\section{Literatur}

1. Ajay VS, Jindal D, Roy A et al (2016) Development of a smartphone-enabled hypertension and diabetes mellitus management package to facilitate evidence-based care delivery in primary healthcare facilities in India: the mPower heart project.J Am Heart Assoc 5:e4343

2. Asada HH, Shaltis P, Reisner A et al (2003) Mobile monitoring with wearable photoplethysmographic biosensors. IEEE Eng Med Biol Mag 22:28-40

3. Attia ZI, Kapa S, Lopez-Jimenez Fet al (2019) Screening for cardiac contractile dysfunction using an artificial intelligence-enabled electrocardiogram. Nat Med 25:70-74

4. Attia ZI, Noseworthy PA, Lopez-Jimenez F et al (2019) An artificial intelligence-enabled ECG algorithm for the identification of patients with atrial fibrillation during sinus rhythm: a retrospective analysis of outcome prediction. Lancet 394:861-867

5. Braun T, Spiliopoulos S, Veltman C et al (2020) Detection of myocardial ischemia due to clinically asymptomatic coronary artery stenosis at rest using supervised artificial intelligence-enabled vectorcardiography - a five-fold cross validation of accuracy. JElectrocardiol 59:100-105

6. Chan J, Rea T, Gollakota S et al (2019) Contactless cardiac arrest detection using smart devices. NPJ Digit Med 2:52

7. Curcio A, de Rosa S, Sabatino J et al (2016) Clinical usefulness of a mobile application for the appropriate selection of the antiarrhythmic device in heart failure. Pacing Clin Electrophysiol 39:696-702

8. Dorr M, Nohturfft V, Brasier N et al (2019) The WATCH AF trial: smartWATCHes for detection of atrial fibrillation. JACC Clin Electrophysiol 5:199-208

9. Drescher FS, Chandrika S, Weir ID et al (2011) Effectiveness and acceptability of a computerized decision support system using modified Wells criteria for evaluation of suspected pulmonary embolism. Ann Emerg Med 57:613-621

10. Evans RS, Kfoury AG, Horne BD et al (2017) Clinical decision support to efficiently identify patients eligible for advanced heart failure therapies. J Card Fail 23:719-726

11. Groenhof TKJ, Asselbergs FW, Groenwold RHH et al (2019) The effect of computerized decision support systems on cardiovascular risk factors: a systematic review and meta-analysis. BMC Med Inform Decis Mak 19:108

12. Guo $Y$, Wang H, Zhang $H$ et al (2019) Mobile photoplethysmographic technology to detect atrial fibrillation. J Am Coll Cardiol 74:2365-2375

13. Indolfi C, Sabatino J, De Rosa S et al (2017) Description and validation of TAVlapp: a novel mobile application for support of physicians in the management of aortic stenosis-management of aortic stenosis with TAVlapp. Biomed Res Int 2017:9027597

14. Luo H, Yang D, Barszczyk A et al (2019) Smartphone-based blood pressure measurement using transdermal optical imaging technology. Circ Cardiovasc Imaging 12:e8857

15. Mairesse GH, Moran P, Van Gelder IC et al (2017) Screening for atrial fibrillation: a European Heart Rhythm Association (EHRA) consensus document endorsed by the Heart Rhythm Society (HRS), Asia Pacific Heart Rhythm Society (APHRS), and Sociedad Latinoamericana de Estimulacion Cardiaca y Electrofisiologia (SOLAECE). Europace 19:1589-1623

16. Maor E, Sara JD, Orbelo DM et al (2018) Voice signal characteristics are independently associated with coronary artery disease. Mayo Clin Proc 93:840-847

17. Maor E, Perry D, Mevorach D et al (2020) Vocal Biomarker is associated with hospitalization and mortality among heart failure patients. J Am Heart Assoc 9:e13359

18. Newzoo (2018) Top countries by smartphone penetration \& users. https://newzoo. com/insights/rankings/top-countries-bysmartphone-penetration-and-users/. Zugegriffen: 25 . Mai 2020

19. O'sullivan JW, Grigg S, Crawford W et al (2020) Accuracy of smartphone camera applications for detecting atrial fibrillation: A systematic review and meta-analysis. JAMA Netw Open 3:e202064

20. Perez MV, Mahaffey KW, Hedlin Hetal (2019) Largescale assessment of a smartwatch to identify atrial fibrillation. NEngl J Med 381:1909-1917

21. Radin J, Wineinger P, Topol Eet al (2020) Harnessing wearable device data to improve state-level realtime surveillance of influenza-like illness in the USA: a population-based study. Lancet Digit Health 2:e85-93

22. Raja AS, Ip IK, Prevedello LM et al (2012) Effect of computerized clinical decision support on the use and yield of CT pulmonary angiography in the emergency department. Radiology 262:468-474

23. Seshadri DR, Bittel B, Browsky D et al (2020) Accuracy of Apple Watch for detection of atrial fibrillation. Circulation 141:702-703

24. Stehlik J, Schmalfuss C, Bozkurt B et al (2020) Continuous wearable monitoring analytics predict heart failure hospitalization: the LINK-HF multicenter study. Circ Heart Fail 13:e6513

25. Sutton RT, Pincock D, Baumgart DC et al (2020) An overview of clinical decision support systems: benefits, risks, and strategies for success. NPJ Digit Med 3:17

26. Thorup C, Hansen J, Gronkjaer M et al (2016) Cardiac patients' walking activity determined by a step counter in cardiac telerehabilitation: data from the intervention arm of a randomized controlled trial. J Med Internet Res 18:e69 


\section{Schwerpunkt}

27. Tomlinson S, Behrmann S, Cranford J et al (2018) Accuracy of smartphone-based pulse oximetry compared with hospital-grade pulse oximetry in healthy children. Telemed JE Health 24:527-535

28. Vandenberk T, Stans J, Mortelmans C et al (2017) Clinical validation of heart rate apps: mixedmethods evaluation study. JMIR Mhealth Uhealth 5:e129

29. Wasserlauf J, You C, Patel Ret al (2019) Smartwatch performance for the detection and quantification of atrial fibrillation. Circ Arrhythm Electrophysiol 12:e6834

30. Werhahn SM, Dathe H, Rottmann T et al (2019) Designing meaningful outcome parameters using mobile technology: a new mobile application for telemonitoring of patients with heart failure. ESC Heart Fail 6:516-525

31. Yan BP, Lai WHS, Chan CKY et al (2018) Contactfree screening of atrial fibrillation by a smartphone using facial pulsatile photoplethysmographic signals. J Am Heart Assoc 7:e8585 